\title{
Tendances pour la pisciculture européenne de demain : cages au large, systèmes en eau recirculée et systèmes intégrés
}

Jean-Paul Blancheton ${ }^{1}$

Pierre Bosc ${ }^{2}$

Jérôme Marie-Édouard Hussenot ${ }^{3}$

Emmanuelle Roque d'Orbcastel ${ }^{1}$

David Romain ${ }^{4,5}$

${ }^{1}$ Institut français de recherche pour l'exploitation de la mer

Station de Palavas

Chemin de Maguelone

34250 Palavas

France

$<$ jpblanch@ifremer.fr>

$<$ emmanuelle.roque@ifremer.fr>

${ }^{2}$ Association réunionnaise

pour le développement de l'aquaculture

$\mathrm{RN} 1$

Les Sables

97427 l'Étang-Salé

La Réunion

<bosc.arda@wanadoo.fr>

${ }^{3}$ Institut français de recherche

pour l'exploitation de la mer

AGSAE

Station de Bouin

Polder-des-Champs

85330 Bouin

France

<Jerome.Hussenot@ifremer.fr>

${ }^{4}$ Institut national des sciences appliquées de Lyon

66, boulevard Niels-Bohr

69100 Villeurbanne

France

<romain.arda@wanadoo.fr>

${ }^{5}$ Laboratoire de mécanique des fluides et d'acoustique (LMFA-Insa)

Ecole centrale de Lyon

36, avenue Guy-de-Collongue

69134 Écully cedex

France

\section{Résumé}

Au cours des dernières décennies, la pisciculture européenne a été principalement fondée sur des systèmes intensifs dévolus à une production monospécifique. Dans la plupart des pays, la compétition avec d'autres activités littorales interdit actuellement l'accès de l'aquaculture marine à de nouveaux sites. La prise en compte de cette contrainte l'a conduite à se tourner vers des systèmes de production en cages au large ou en recirculation à terre dans des zones peu convoitées. De plus, dans le contexte actuel de la diminution des ressources en eau et de réduction de l'impact des activités sur l'environnement, les systèmes en eau recirculée présentent l'avantage de limiter la consommation en eau et de faciliter le traitement des rejets. Les systèmes intégrés permettent, quant à eux, de valoriser les nutriments rejetés par les poissons par des coproductions d'algues, de mollusques ou de poissons qui s'en nourrissent et de rejeter une eau épurée. Cet article présente quelques aspects du développement de ces trois types de systèmes d'élevage vers lesquels s'oriente actuellement la pisciculture : les cages au large, les systèmes en eau recirculée et les systèmes intégrés.

Mots clés : cage à poisson ; Europe ; gestion intégrée ; installation au large des côtes ; pisciculture.

Thèmes : pêche et aquaculture ; production animale.

\section{Abstract \\ The 'new' European fish culture systems: Recirculating systems, offshore cages, integrated systems}

Over the last few decades, European fish culture was developed mainly as a intensive monospecific activity. In most countries, marine aquaculture cannot continue to develop on the shore line where it first settled due to high competition with other activities. It has now to move either offshore or into recirculation systems located in less occupied spaces. Fresh water availability and impact of fish production farms on the environment are strongly restraining the development of the activity, which is even declining in some countries such as France. Recirculation systems make it possible to decrease water consumption and facilitate waste treatment. Their economical feasibility is being demonstrated and they offer an alternative to developing fish farms. Whatever the rearing system, nutrients of feed are partially converted into fish flesh and most of them are thrown back as wastes into the environment. Integrated systems make it possible to convert such nutrients into algae, molluscs or fish and to release purified water. This article presents some aspects of the development of three types of rearing systems which are increasingly used in northern Europe: offshore cage culture, recirculation systems and integrated systems.

Key words: Europe; fish cages; integrated management; offshore structures; pisciculture.

Subjects: animal production; fishing and aquaculture.

Tirés à part : J.-P. Blancheton 
ace à la stagnation des prises de pêche, la pisciculture apparât aujourd'hui comme une alternative incontournable pour répondre à une demande en poisson qui croît régulièrement. Les contraintes qui pèsent actuellement sur le développement de cette activité (compétitions pour l'espace, disponibilité en protéines et en huiles pour fabriquer l'aliment, coût de l'énergie) conduisent à reconsidérer les systèmes de production traditionnellement utilisés. Au cours des dernières décennies, la pisciculture du type monoculture intensive en mer ou à terre, en système ouvert ou en système en recirculation, qui s'est développée dans les pays du Nord, a été confrontée à des conflits d'usage (Milstein, 2005) essentiellement liés à l'utilisation de l'eau (pisciculture continentale) et à l'occupation de l'espace (pisciculture marine). La difficulté d'accès aux sites dans les zones littorales constitue un frein majeur au développement de la pisciculture marine (conflits d'utilisation, en particulier dans les zones touristiques). L'aquaculture doit s'éloigner des côtes pour poursuivre son développement. Cette orientation a été prise depuis plusieurs années dans des pays où la pisciculture revêt une grande importance (Japon, Corée, Turquie, etc.) et dans la plupart des régions ultrapériphériques européennes (Canaries, Madère, Réunion, etc.). Les cages au large permettent de lever l'obstacle de l'espace disponible, d'éloigner le système d'élevage des zones de conflits et de minimiser l'impact des rejets sur le milieu; en s'éloignant des côtes, la profondeur et l'hydrodynamisme des sites augmentent, ce qui permet une meilleure dilution des rejets dans la colonne d'eau.

Une autre solution consiste à développer les systèmes d'élevage à terre, en retrait des zones côtières, dans des systèmes intensifs en recirculation qui n'utilisent que peu d'eau et peu d'espace (Piedrahita, 2003 ; Blancheton et al., 2004 ; Sindilariu, 2007). Ces systèmes en eau recirculée de prendre en compte les exigences des poissons vis-à-vis de la qualité de l'eau et de traiter les rejets produits afin d'en limiter l'impact sur l'environnement (Bergheim et Brinker, 2003). Différents types de systèmes en eau recirculée sont envisageables selon le type de poissons produit (Blancheton et al., 2007 ; Crab et al., 2007). Leur viabilité économique a été établie pour l'élevage de larves et de géniteurs d'espèces marines, ainsi que pour le grossissement d'espèces d'eau douce chaude, les économies d'énergie de chauffage permettant de compenser en partie le coût de l'investissement lié à la zone de traitement de l'eau recirculée (Blancheton, 2000). Depuis quelques années, des systèmes en eau recirculée à faible coût ont été adaptés au cas du grossissement des espèces d'eau douce froide à plus faible valeur ajoutée (Roque d'Orbcastel, 2008).

Quel que soit le système d'élevage, seulement environ $25 \%$ de l'azote et $30 \%$ du phosphore de l'aliment sont intégrés dans la chair des poissons (Hargreaves, 1998 ; Lupatsch et Kissil, 1998). Les nutriments non digérés et les catabolites issus du métabolisme digestif sont rejetés dans l'environnement sous forme de matières dissoutes (azote ammoniacal, urée et orthophosphates principalement) et de matières solides (fèces et aliment non consommé en cas de gaspillage alimentaire). La polyculture, ou plus exactement la co-culture (Billard, 2003), repose sur l'utilisation des rejets issus d'un premier maillon d'élevage comme source de nutriments utilisables par le maillon suivant (Lightfoot et al., 1993; Yan et al., 1998). Il devient alors possible de valoriser les nutriments issus des rejets d'un élevage par des productions aquacoles supplémentaires. Ces systèmes d'élevage sont appelés "systèmes intégrés" ou encore "systèmes multitrophiques ", et les rendements d'utilisation des nutriments de l'aliment sont alors considérablement améliorés.

L'objectif de cet article est de présenter quelques aspects de ces trois types de systèmes d'élevage vers lesquels s'oriente actuellement la pisciculture dans les pays du Nord : les cages au large, les systèmes en eau recirculée et les systèmes intégrés.

\section{Exemple de développement de cages au large : la pisciculture marine à La Réunion}

En 2000, un projet visant à mettre au point un modèle de cages flottantes adapté aux conditions de l'offshore tropical a été initié à La Réunion pour produire de l'ombrine (Sciaenops ocellatus). Le programme a été lancé dans le cadre d'une opération pilote réalisée en collaboration avec un producteur local et un constructeur de cages franco-espagnol.

\section{Mise au point d'un premier système de cages au large}

Le premier facteur de risque des cages au large concerne les effets des houles et des courants extrêmes sur la tenue des structures d'élevage. À La Réunion, les houles australes, caractérisées par de fortes amplitudes (jusqu'à $12 \mathrm{~m}$ au déferlement), sont susceptibles de générer des courants orbitaux pouvant être ponctuellement violents (3 à $4 \mathrm{~m} / \mathrm{s}$ ) durant généralement de 24 à 72 heures. Les houles cycloniques, moins fréquentes, génèrent des états d'agitation extrêmement violents pouvant durer plus de 72 heures.

L'adaptation des cages aux conditions offshore a consisté à dimensionner les amarrages en intégrant des amortisseurs de houles à contrepoids et à concevoir un dispositif d'immersion pour échapper aux mers de vents cycloniques (figure 1). L'amarrage est une pièce maîtresse des cages qui maintient en place le support d'élevage et absorbe une grande partie des forces subies par les structures. Il doit résister aux efforts conjugués de la houle, du courant et du vent et permettre une certaine liberté de mouvement pour laisser le support se déplacer sous l'effet des vagues afin de diminuer les efforts. Il peut être assimilé à un ressort qui doit être dimensionné afin de se déformer, tout en étant constamment en tension, et ne pas entraîner l'immersion des supports flottants. Il n'existe pas de modèle disponible pour élaborer des plans d'amarrages théoriques susceptibles d'offrir les meilleures conditions de résistance aux efforts. Les contrepoids (masse unitaire de 250 à $500 \mathrm{~kg}$ ) jouent un rôle déterminant dans l'amortissement des efforts exercés sur le dispositif. Ce type d'amarrage est en tension permanente, mais il n'accepte pas les forts marnages et nécessite un positionnement précis des ancrages.

L'immersion des structures constitue l'unique solution pour réduire les contraintes générées par les très fortes houles. La technique d'immersion retenue est basée sur le ballastage des cages réalisé grâce à un dispositif de vannes ins- 


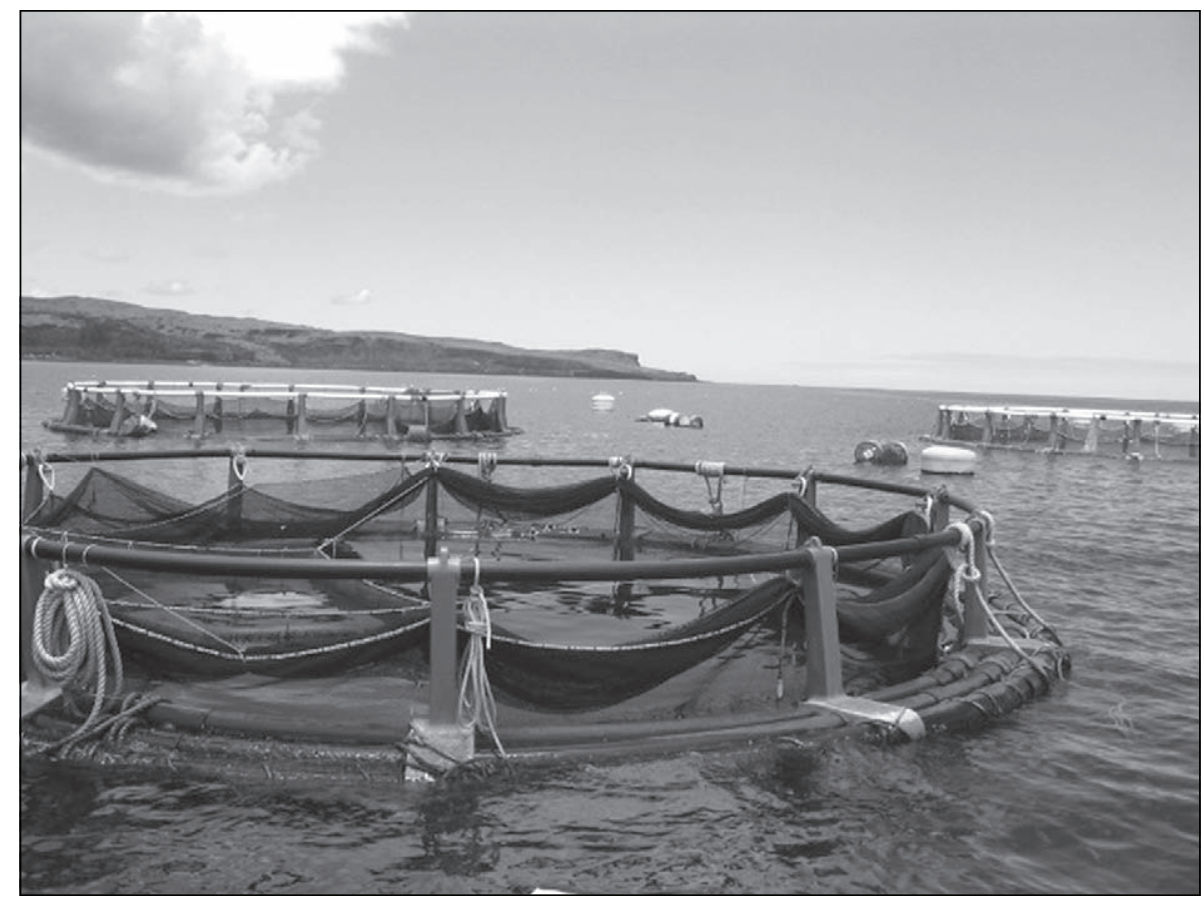

Figure 1. Modèle de cage circulaire.

Figure 1. Circular cage.

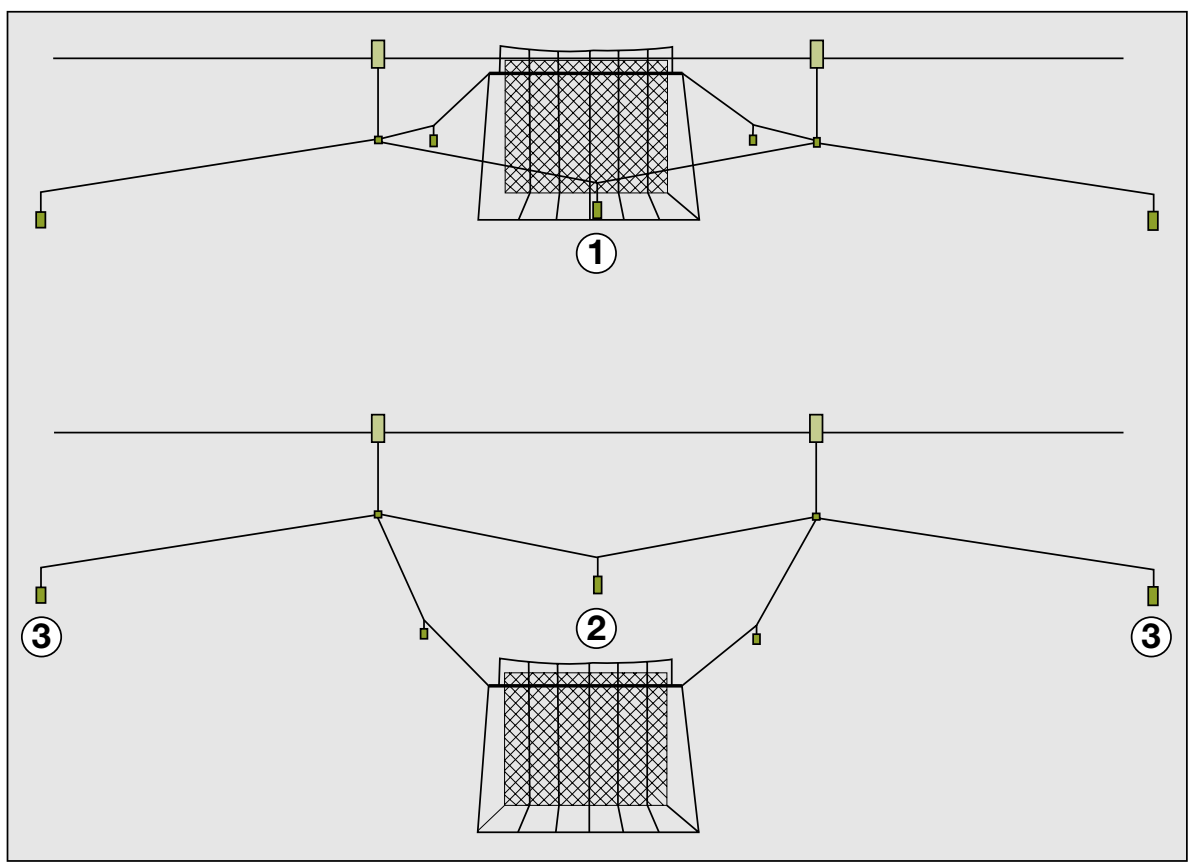

Figure 2. Fonctionnement du dispositif d'immersion.

Figure 2. Operation of the structure immersion.

1 : cage avec son filet de protection ; 2 : flotteur ; 3 : contrepoids.

tallées sur la structure flottante et qui permet de remplir d'eau les anneaux de polyéthylène (figure 2). Immergée (à
$15 \mathrm{~m}$ de profondeur), la structure est retenue par les bouées de surface. Le retour à la surface se fait en envoyant de l'air dans les anneaux (compresseur embarqué ou bouteilles de plongée). Une fois immergé, le point faible du dispositif est la tenue des bouées de surface qui doivent supporter les fortes contraintes.

Les cages au large se comportent comme des dispositifs de concentration de petits pélagiques qui attirent les prédateurs. Ces derniers peuvent occasionner des dégâts, et il est donc indispensable d'installer des filets de protection autour des cages, ce qui pose des problèmes de résistance des dispositifs d'amarrage liés aux efforts de traînée induits. Le dispositif de protection contre les prédateurs est constitué de filets de chaluts. Très résistants, ils offrent un bon compromis entre coût et souplesse d'exploitation et sont lestés par un anneau de fond sur lequel peut être amarré le filet d'élevage. Le principal inconvénient réside dans la prise au courant du filet. Sa présence impose donc un surdimensionnement des amarrages pour résister aux efforts de traînée qu'il engendre.

\section{Premiers résultats obtenus à La Réunion et améliorations possibles}

Après cinq ans d'exploitation, le système de cages offshore a démontré son adaptation au contexte local pour une échelle d'exploitation semi-industrielle (60 à $70 \mathrm{t}$ d'ombrines par an, 38 à $45 \mathrm{~m}^{3}$ de cage par tonne produite). Les résultats zootechniques ont confirmé ceux de Bosc et Gaumet (2002) avec un cycle de huit mois pour le grossissement de poissons jusqu'à environ $1 \mathrm{~kg}$ (charge $20 \mathrm{~kg} / \mathrm{m}^{3}$; IC ${ }^{1}$ de 1 1,5 ; survie moyenne de $90 \%$ ). Les cages, installées sur des fonds de 35 à $45 \mathrm{~m}$, ont subi en 2007 l'une des plus violentes houles cycloniques des 50 dernières années (creux de plus de $8 \mathrm{~m}$ pendant plus de 24 heures). Immergées dès l'alerte par $15 \mathrm{~m}$ de fond, elles ont montré une bonne résistance et ont permis un maintien du cheptel sans mortalité anormale (taux de $3 \%$ pendant 15 jours). Deux études (Bosc, 2004; Cam, 2005) ont montré que la production minimale pour amortir les coûts d'exploitation est de 120 à 140 t/ an. Quelques modifications permettraient facilement d'adapter ce système à des projets plus ambitieux. Pour améliorer sa sécurisation vis-à-vis du risque cyclonique, un programme d'évaluation des

\footnotetext{
${ }^{1}$ IC : indice de conversion alimentaire ( $\mathrm{kg}$ d'aliment par kg de poisson produit).
} 
points faibles du dispositif d'amarrage a été initié en 2006 en partenariat avec l'Insa de Lyon (Romain, 2007). Des progrès sont possibles en réduisant les forces de traînée des filets antiprédateurs et en optimisant l'hydrodynamisme des bouées de surface.

\section{Systèmes}

\section{en eau recirculée}

En raison des contraintes environnementales de plus en plus fortes, des élevages en système en eau recirculée se développent. Dans ce type de système, où les poissons sont élevés à haute densité, les besoins en eau neuve sont divisés par un facteur 10 à 100 par rapport à un système d'élevage en circuit ouvert classique ; on parle du " niveau de fermeture " (exprimé en mètres cubes d'eau consommée par $\mathrm{kg}$ de poisson produit) du système. Le volume des rejets est diminué par le même facteur, et leur concentration est plus élevée, ce qui facilite leur traitement. Le degré de fermeture d'un système recirculé dépend de l'espèce élevée et du degré de sophistication du système de traitement de l'eau. Celui-ci combine des procédés physicochimiques (filtration mécanique, échange liquide/gaz, contrôle de la température et du $\mathrm{pH}$ ) et biologiques (biofiltration aérobie et anaérobie). La plupart du temps, les rejets sont traités dans un circuit annexe. Durant les 20 dernières années, ce type de système d'élevage a été développé dans les pays d'Europe du Nord pour le grossissement d'espèces d'eau douce (anguilles et poissons-chats africains) et dans les pays d'Europe du Sud pour la production d'alevins et le prégrossissement d'espèces marines (bar, daurade et turbot). Le système en eau recirculée est de plus en plus utilisé pour le grossissement de poissons d'eau douce ou d'eau de mer, et les exploitations permettent maintenant de produire plusieurs centaines de tonnes de poissons par an.

\section{Types de systèmes en eau recirculée couramment rencontrés}

Les systèmes en eau recirculée assurent le maintien d'un milieu d'élevage de qualité constante et modulable, avec des variations d'amplitudes faibles et lentes. En même temps, ils permettent des économies notables d'énergie de chauffage (Blancheton et al., 1997). Dans ces systèmes, la mise en place de mesures de biosécurité est possible, consistant à combiner un contrôle sanitaire des géniteurs (détection précoce des pathogènes) et un contrôle optimal de la qualité de l'eau (en entrée, en sortie et dans le système). Cette approche permet d'éviter toute propagation de maladies d'une exploitation à l'autre ou au sein de l'exploitation et constitue un élément essentiel de la rentabilité de ces systèmes (Breuil et al., 2001). L'association de ces systèmes d'élevage avec l'amélioration des méthodes de nourrissage des alevins et de la composition de l'aliment a été la clé qui a permis l'émergence, au début des années 1990, d'une production viable d'alevins dans la plupart des écloseries européennes (Blancheton, 2000).

Les systèmes en eau recirculée ont été développés à l'échelle commerciale en Europe, particulièrement pour le grossissement de poissons d'eau douce (Heinsbroek et Kamstra, 1990 ; Verreth et Eding, 1993) et marins, avec une capacité de production moyenne d'environ $100 \mathrm{t}$ /an (Blancheton, 2000). Désormais, la technologie est maîtrisée, et des applications commerciales ont démontré son applica- bilité. À titre d'exemple, 90 exploitations fonctionnent en recirculation aux PaysBas et produisent environ 9000 t/an. Des systèmes pilotes (plusieurs tonnes par an) ont été utilisés pour l'élevage d'espèces telles que l'ombrine (Sciaenops ocellatus) ou le silure (Silurus glanis) en France ou la sole (Solea solea) et le sandre (Stizostedion lucioperca) aux Pays-Bas. Quel que soit le concept, les systèmes de traitement de l'eau et les bassins d'élevage doivent être adaptés aux besoins des poissons, qui diffèrent d'une espèce à l'autre. Tous les systèmes en eau recirculée sont conçus selon le même schéma de base (Bovendeur et al., 1987 ; Blancheton et al., 1997 ; Kamstra et al., 1998) (figure 3). L'eau des bassins d'élevage est d'abord filtrée mécaniquement (maille de 40 à $100 \mu \mathrm{m}$ ) pour séparer les matières en suspension (MES). L'eau passe ensuite dans un filtre biologique où l'ammoniaque est oxydée par nitrification. La configuration et la gestion du filtre biologique jouent un rôle essentiel visà-vis de l'efficacité de la nitrification. L'élimination du dioxyde de carbone issu de la respiration du poisson est assurée par une colonne de dégazage (ou dans un filtre biologique à ruissellement) ventilée. L'oxygène est directement injecté dans l'eau alimentant les bassins d'élevage.

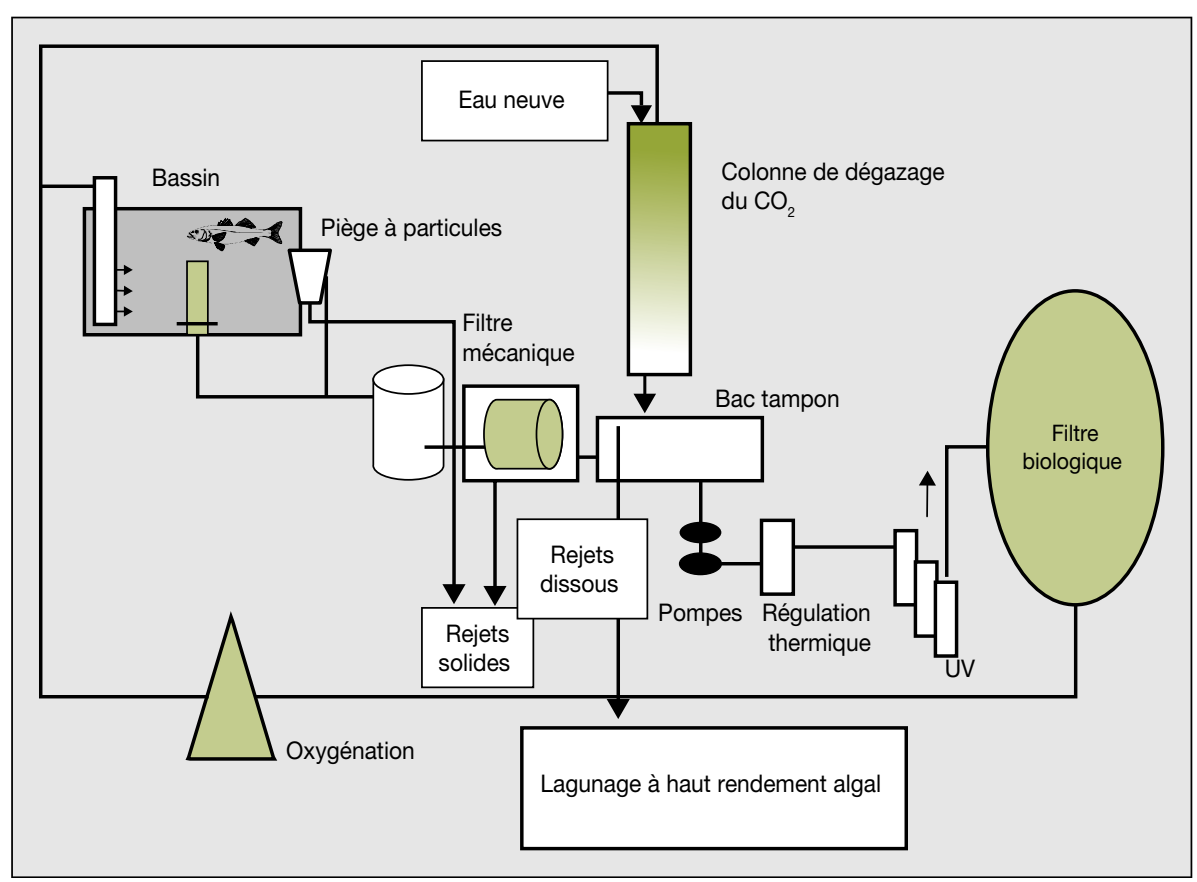

Figure 3. Schéma d'un système en eau recirculée.

Figure 3. Scheme of a recirculation system. 


\section{Principales technologies des systèmes développés en eau douce et en eau de mer}

Les poissons d'eau douce, tels que le poisson-chat africain (Clarias gariepinus) ou l'anguille, (Anguilla sp) ne sont pas exigeants quant à la qualité de l'eau. Cela permet de mettre en ouvre des débits de recirculation réduite et des circuits très fermés. Dans ce type d'installation, l'eau peut subir une simple décantation avant de passer dans un filtre à ruissellement ventilé qui associe nitrification, élimination du gaz carbonique et refroidissement si nécessaire. Parfois, un filtre dénitrifiant alimenté en carbone (fèces de poisson, sucre ou alcool) permet de diminuer la concentration en nitrates de l'eau et donc l'apport d'eau neuve nécessaire. L'effluent sortant du filtre dénitrifiant est réinjecté dans le système d'élevage via un filtre à tambour. Dans les systèmes en eau recirculée sans dénitrification, c'est l'apport d'eau neuve qui permet de maintenir la concentration en nitrates en dessous de la valeur seuil recommandée. Dans les systèmes les plus récents, une unité de floculation (déphosphatation) combinée à un filtre à bande est installée pour traiter les matières solides de l'effluent final qui peut alors être rejeté directement dans le milieu.

La matière organique qui n'est pas éliminée lors de la filtration mécanique peut être dégradée par les bactéries en substances organiques dissoutes (Léonard et al., 2002; Chen et al., 2006 ; Michaud et al., 2006).

Les poissons marins sont plus sensibles que les poissons d'eau douce à l'accumulation de ces sous-produits, ce qui nécessite un niveau de fermeture des systèmes plus limité. L'équilibre bactérien est très important, surtout pour les stades précoces: une population bactérienne stable évite les blooms monospécifiques de bactéries, qui peuvent constituer une menace pour les poissons. Le contrôle de ces populations est souvent effectué grâce à une unité de désinfection par les UV qui en réduit la concentration et inactive les principales bactéries potentiellement pathogènes (Léonard et al., 2000). Dans un système fonctionnant en routine, la consommation d'oxygène des bactéries est du même ordre de grandeur que celle des poissons. Environ, la moitié de la consommation bactérienne est due à des bactéries hétérotrophes, qui métabo- lisent les petites particules restant en suspension dans l'eau après filtration. Ces bactéries hétérotrophes concurrencent les autotrophes pour la disponibilité en surface de fixation et en oxygène et provoquent une diminution du rendement d'oxydation de l'ammoniaque (Bovendeur et al., 1990) et la création de conditions défavorables ( $\mathrm{pH}$ bas et création de produits de dégradation dissous).

\section{Perspectives et conclusion}

Les systèmes recirculés en eau de mer et en eau douce, qui différaient principalement au niveau des outils de traitement de l'ammoniaque et du $\mathrm{CO}_{2}$, sont maintenant de plus en plus proches, car la salinité est un facteur secondaire : c'est le type de poisson élevé et le stade d'élevage qui sont les éléments prioritaires à prendre en compte pour concevoir un système. Par ailleurs, il est intéressant de noter que, quel que soit le système, les besoins en énergie sont proches (tableau 1). Dans le contexte actuel, il est important de diminuer la consommation d'énergie par kilogramme de poisson produit. Des essais réalisés sur des systèmes en eau recirculée s'inspirant de ceux mis en œuvre pour les salmonidés au Danemark ont montré que la consommation d'énergie peut être réduite (Roque d'Orbcastel, 2008). Ces systèmes sont intermédiaires entre les systèmes ouverts et les systèmes en eau recirculée traditionnels, avec une consommation d'eau neuve d'environ $8 \mathrm{~m}^{3} / \mathrm{kg}$ d'aliment distribué et un traitement de l'eau simplifié. Dans ces systèmes, les performances et le bien-être des poissons sont similaires à ceux de poissons élevés en circuit ouvert (Roque d'Orbcastel, 2008).

Alors que pour toutes les espèces la croissance en système en eau recirculée est meilleure qu'en circuit ouvert en début de grossissement, une réduction de la croissance a été observée dans les systè- mes en eau recirculée marins. Son intensité dépend de l'espèce et apparait audelà d'un poids moyen qui, lui aussi, dépend de l'espèce. Des composés dissous non identifiés, dont la concentration augmente quand la quantité d'eau renouvelée par kilogramme d'aliment décroît, sont probablement responsables de ce phénomène. Des recherches sont actuellement en cours afin d'identifier la cause du problème ainsi que des solutions pour le résoudre. Le traitement des effluents d'élevages est une priorité dans la perspective de l'application de la directive cadre Eau européenne en 2015. Ce traitement est facilité dans le cas des systèmes en eau recirculée par la réduction des débits et la concentration des rejets. Des études ont démontré que le lagunage à haut rendement algal est adapté au traitement des effluents de systèmes recirculés marins en eau recirculée (Pagand et al., 2000) et permettent une réutilisation dans le circuit de l'effluent après traitement (Deviller et al., 2002).

\section{Systèmes intégrés en aquaculture}

La polyculture en étang est le système d'aquaculture le plus ancien et toujours le plus pratiqué dans le monde. Il s'agit de systèmes d'élevage extensifs à semiintensifs, associant plusieurs espèces de façon à occuper les différentes niches écologiques du bassin d'élevage pour optimiser la production. Les déchets produits par une espèce peuvent être valorisés par d'autres. Dans les pays du Sud, l'aquaculture est même parfois étroitement associée aux productions agricoles dont elles utilisent les déchets organiques. Dans les pays du Nord, la monoculture aquacole inten-

\section{Tableau 1. Caractéristiques de systèmes en eau recirculée pour une production annuelle de 100 tonnes d'anguille, de bar et turbot.}

Table 1. Characteristics of recirculation systems for a $100 \mathrm{MT}$ annual production of eel, seabass and turbot.

\begin{tabular}{lccc}
\hline Caractéristiques & Anguille & Bar & Turbot \\
\hline Poids individuel $(\mathrm{g})$ & $0,25-150$ & $10-350$ & $10-1000$ \\
Productivité $\left(\mathrm{kg} / \mathrm{m}^{2}\right.$ par an) & $200-300$ & 200 & 70 \\
Biomasse finale en stock $\left(\mathrm{kg} / \mathrm{m}^{2}\right)$ & 114 & 100 & 50 \\
Énergie $(\mathrm{kWh} / \mathrm{kg}$ par aliment) & 7 & 6,5 & 6,7 \\
Productivité de la main-d'oeuvre (t/an/personne) & 70 & 95 & 50 \\
\hline
\end{tabular}


sive a posé des problèmes de pollution des rivières et des eaux littorales. L'enrichissement des eaux rejetées repose sur le fait qu'une faible part de l'azote et du phosphore, introduits dans le système d'élevage sous la forme d'aliment composé, est intégrée dans la chair des poissons (Hall et al., 1992; Hargreaves, 1998 ; Holby et Hall, 1991 ; Lupatsch et Kissil, 1998). Si aucune précaution n'est prise, les nutriments non métabolisés sont rejetés dans l'environnement sous forme de matière dissoute et solide.

\section{Principe \\ des systèmes intégrés}

Le concept du système intégré a été décrit par Schneider et al. (2005) qui identifient cinq modules où s'opère la conversion des flux de nutriments azotés et phosphorés (figure 4). Le premier module est l'élevage intensif principal, qui transforme l'aliment en poisson. Le module 2 sert au traitement primaire des rejets provenant du module 1. Il peut comprendre un bassin de décantation ou un filtre mécanique à tambour, ou tout autre procédé pouvant diminuer la concentration en rejets solides. Le module 3 est utilisé pour la conversion des rejets dissous $\left(\mathrm{CO}_{2}, \mathrm{~N}, \mathrm{P}\right)$ par la photosynthèse en végétaux (microalgues, macroalgues, ou macrophytes), qui peuvent être utilisés par des herbivores. Le module 4 est dévolu à la conversion des rejets en biomasse bactérienne, qui peut constituer un apport substantiel de protéines dans l'alimentation. Le module 5 permet de convertir les détritus et les bactéries par des organismes invertébrés comme les polychètes (Nereis $s p p$.), utilisables pour l'alimentation des poissons. Les systèmes photosynthétiques en suspension décrits par Hargreaves (2006) correspondent à une forme particulière de système intégré, développée en bassin artificiel ou étang. Elle concerne les systèmes d'élevage "eau verte " ou "échange zéro ", dans lesquels le renouvellement d'eau est réduit au minimum. La matière organique et les nutriments rejetés par l'élevage sont digérés et assimilés par des bactéries et/ou par des organismes photosynthétiques, ce qui nécessite de maintenir les particules en suspension et d'aérer le milieu d'élevage. Ces systèmes sont plus particulièrement développés en eau douce pour le tilapia ou le poissonchat et en eau de mer pour les crevettes pénéides.

En choisissant plusieurs espèces occupant différentes niches, il est possible de construire un système d'élevage dans lequel les différentes cultures interagissent entre elles, ce qui aura pour effet de

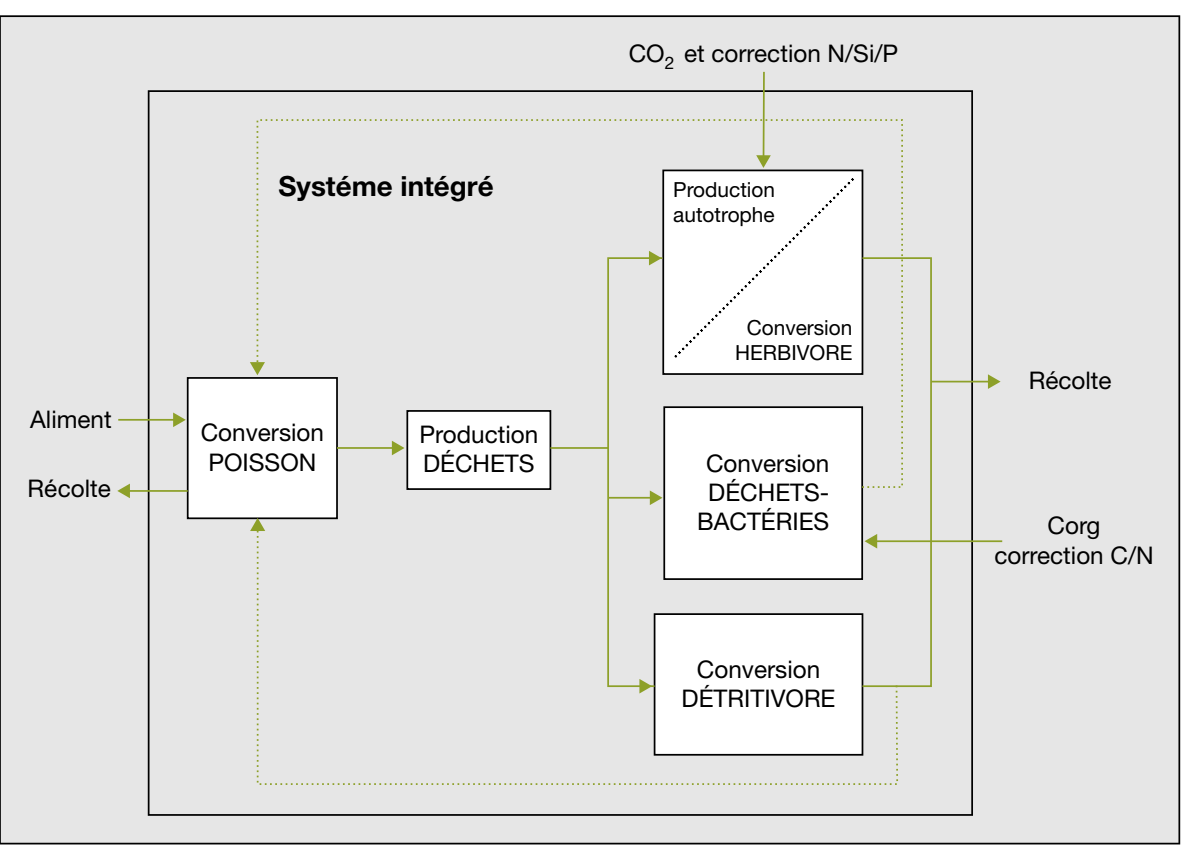

Figure 4. Concept du système intégré.

Figure 4. Concept of the integrated system.

Les lignes pointillées correspondent à des flux possibles dans le futur, les lignes pleines aux flux des systèmes existants (source projet ZAFIRA et Schneider et al., 2005). diminuer les rejets de carbone, d'azote et de phosphore de la culture principale, grâce à l'activité d'espèces autotrophes ou hétérotrophes. La chaîne trophique simplifiée mise en place conduira à une production extensive complémentaire d'animaux herbivores, détritivores ou carnivores, diversifiant la production aquacole de l'exploitation (Shpigel et Neori, 1996 ; Schaber, 1997 ; Hussenot et al., 1998; Neori et al., 2004; Hussenot, 2006). La réduction de l'impact de l'activité sur l'environnement aquatique est alors très significative. D'après une récente synthèse effectuée sur six études de systèmes intégrés par Schneider et al. (2005), 60-85\% de l'azote et 50-90\% du phosphore peuvent être retenus par ce type de système, alors que la seule conversion en biomasse de poisson ne retient, en monoculture intensive, que $14-30 \%$ de l'azote et $20-42 \%$ du phosphore apporté par l'aliment.

\section{Quelques exemples de systèmes intégrés}

L'idée de culture intégrée pour développer une aquaculture respectueuse de l'environnement s'est développée dans la littérature scientifique depuis environ trois décennies dans les pays du Nord (Gordin et al., 1981 ; Chopin et al., 2001 ; Brune et al., 2003; Hussenot et Shpigel, 2003), alors que la polyculture était déjà une pratique culturale profondément ancrée dans les pays du Sud. Elle était décrite depuis le $\mathrm{V}^{\mathrm{e}}$ siècle avant J.-C. dans le traité de pisciculture de Fan Li (Billard, 2000) pour la gestion des étangs d'eau douce en Chine et depuis le Xv ${ }^{\mathrm{e}}$ siècle en lagunes d'eau salée ou saumâtre d'Indonésie, d'Afrique de l'Ouest ou d'Hawaï (Brezski et Newkirk, 1997). Aujourd'hui, de nouveaux systèmes intégrés aquacoles ont été proposés, comme par exemple les associations saumon, macroalgues, moules (Canada, Norvège), macroalgues-ormeaux/oursins-daurades royales (Israël), poissons-chat africains-microalgues-tilapias/carpes (Hongrie), barsmicroalgues-bivalves (France), crevettes pénéides-huîtres-macroalgues (Australie). En eau douce, des systèmes intégrés associant aquaculture et agriculture sont largement répandus (Kumar, 2000 ; Prein, 2002) : traditionnellement riz et poisson au Vietnam, carpe et polyculture en Chine ou au Bangladesh, poisson-chat et poulets en Thailande. Des techniques plus récentes ont été développées comme les cultures hydroponiques de 
légumes en aval d'élevages de poissons tilapias (Rakocy, 1989 ; Seawright et al., 1998). De la même façon, en milieu marin, des essais de démonstration à grande échelle associent à terre des plantes halophytes (salicornes, arbres de mangrove) à la pisciculture marine (Hodges et al., 1993; Brown et al., 1999). De nombreux projets pilotes ont été développés à petite échelle; ainsi, un système pilote industriel de culture associée de saumon (Salmo salar), de laminaire (Laminaria saccharina) et de moule (Mytilus edulis), a été développé en baie de Fundy (Canada) (Chopin et Bastarache, 2002). Dans le but de développer une polyaquaculture de poissons et de macroalgues, des études ont été menées sur les performances de croissance de macroalgues valorisables cultivées sur effluents de pisciculture marine (Lüning et al., 2003). Des élevages de crevettes pénéïdes ont été développés à grande échelle sur les bords de la mer Rouge (Stolte, 2003). L'eau de rejet de ces élevages alimente des élevages secondaires de tilapias et de milkfish qui sont effectués dans les chenaux d'irrigation où transite l'eau de rejet qui alimente des champs d'arbres à mangrove et de salicornes avant d'être rejetée dans des salines où sont produites des artémies. Des recherches ont également porté sur le développement d'une approche générique de systèmes intégrés d'aquaculture marine durable à destination des marchés européens (Hussenot, 2003).

\section{Conclusion et perspectives}

Optimiser la croissance simultanée de différentes espèces qui interagissent entre elles s'avère être une tâche complexe, aussi bien du point de vue technique que du point de vue économique ou sanitaire. Afin de mettre en place progressivement une aquaculture intégrée durable, il apparaît indispensable d'imaginer, de tester et de valider de nouveaux systèmes d'associations d'espèces.

La pisciculture européenne est aujourd'hui confrontée à des contraintes d'ordre réglementaire sur les rejets d'élevage (application de la directive cadre Eau d'ici à 2015), social quant à l'accès aux sites et d'ordre environnemental vis-à-vis de la diminution des ressources en eau. Des solutions techniques existent pour permettre le développement d'une pisciculture plus durable. Des systèmes d'élevage en cages compatibles avec les conditions du large sont en cours de développement et ont d'ores et déjà démontré leur fiabilité. D'autres solutions techniques comme la reconversion de plates-formes pétrolières dans l'Atlantique nord sont actuellement en cours d'étude. Les systèmes en eau recirculée sont utilisés en Europe à échelle industrielle pour la production d'alevins et leur prégrossissement. Des unités de grossissement de grande taille (millier de mètres cube) sont en phase de démarrage sur des espèces comme le bar, la morue ou les salmonidés. L'optimisation de ces systèmes nécessite d'intégrer en parallèle des améliorations techniques et une connaissance approfondie des espèces élevées et de l'environnement bactérien, afin de respecter le bien-être des animaux, la qualité du produit final, tout en minimisant l'impact environnemental de l'activité. Les systèmes intégrés apparaissent comme une voie complémentaire vers une aquaculture plus durable. Ils peuvent permettre l'utilisation de territoires aujourd'hui interdits à l'aquaculture intensive, sans dommages pour l'environnement et en contribuant à la conservation des zones humides et à leur biodiversité. Ce type d'élevage contribue efficacement à améliorer le rendement d'utilisation des intrants, donc à réduire les impacts des élevages sur l'environnement.

\section{Références}

Bergheim A, Brinker A. Effluent treatment for flow through systems and European environmental regulations. Aquac Eng 2003; 27: 61-77.

Billard R. Le Traité de Fan Li $\left(v^{e}\right.$ siècle avant J.-C.) et la pisciculture en Chine. II Pesce 2000; (février) : 54-61.

Billard R. From polyculture to co-culture in fish farming. In: Lee CS, ed. Aquaculture: retrospective and outlook. An aquaculture summit. Manila; Baton Rouge: Asian Fisheries Society and World Aquaculture Society, 2003.

Blancheton J-P. Developments in recirculation systems for Mediterranean fish species. Aquac Eng $2000 ; 22$ : 17-31.

Blancheton J-P, Covès D, Lemarié G. Intensive land-based marine fish aquaculture in closed system, hatchery and ongrowing units: state of the art and prospects. J Jpn Aquac Soc Suisanzoshoku 1997 ; H9: 143-9.

Blancheton JP, Dosdat A, Deslous-Paoli JM. Minimisation des rejets biologiques issus d'élevages de poissons. Dossiers de l'environnement de I'Inra 2004; 26 : 67-78.

Blancheton JP, Piedrahita RH, Eding EH, et al. Intensification of land-based aquaculture production in single pass and reuse systems. In: Aquaculture engineering and environment. SI : sn, 2007.
Bosc P. Contribution à l'étude de la structure des coûts de production de l'ombrine. Programme d'évaluation des perspectives de développement de l'élevage en baie de SaintPaul (La Réunion). SI : ARDA, 2004.

Bosc $P$, Gaumet F. Bilan technique des travaux 1999-2002 sur les systèmes d'élevage en cage en baie de Saint-Paul. SI : ARDA, 2002.

Bovendeur J, Eding EH, Henken AM. Design and performance of a recirculation system for high-density culture of the African catfish, Clarias gariepinus (Burchell 1822). Aquaculture $1987 ; 63: 329-53$.

Bovendeur J, Zwaga AB, Lobee BGJ, Blom JH. Fixed-biofilm reactors in aquacultural water recycle systems: Effect of organic matter elimination on nitrification kinetics. Water Res 1990 ; $24: 207-13$.

Breuil G, Thiery R, Pépin J-F, Blancheton J-P. The control of nodavirus disease in seabass, Dicentrarchus labrax, from breeder to commercial size: toward a new approach of organic aquaculture. Aquaculture Interchange Program (AIP) workshop, Biosecurity in aquaculture production systems: exclusion of pathogens and other undesirables, 23-26 July, 2001, Honolulu, Hawaï (USA). Palavas-lesFlots : Ifremer, 2001.

Brezski V, Newkirk G. Integrated coastal food production systems: a review of current literature. Ocean Coast Manage 1997 ; 34 : 55-71.

Brown JJ, Glenn EP, Fitzsimmons KM, Smith $\mathrm{SE}$. Halophytes for the treatment of saline aquaculture effluent. Aquaculture 1999; 175 : 255-68.

Brune DE, Schwartz G, Eversole AG, Collier JA, Schwedler TE. Intensification of pond aquaculture and high rate photosynthetic systems. Aquac Eng 2003 ; 28 : 65-86.

Cam D. Schéma Directeur de I'aquaculture réunionnaise. Rapport CRPMEM, REPAL, CYATHEA, ADEQUA. 2005.

Chen S, Ling J, Blancheton JP. Nitrification kinetics of biofilm as affected by water quality factors. Aquac Eng 2006 ; 34 : 224-33.

Chopin T, Buschmann AH, Halling C, et al. Integrating seaweeds into marine aquaculture systems: a key toward sustainability. J Phycol $2001 ; 37: 975-86$.

Chopin T, Bastarache S. Finfish, shellfish and seaweed mariculture in Canada. Bull Aquac Assoc Can 2002 ; 102 : 119-24.

Crab R, Avnimelech Y, Defoirdt T, Bossier P, Verstraete W. Nitrogen removal techniques in aquaculture for a sustainable production. Aquaculture $2007 ; 270$ : 1-14.

Deviller G, Blancheton JP, Franco Nava MA, Casellas C. High rate algal pond treatment for water reuse in a marine fish recirculation system. Abstract of a presentation at the World Aquaculture 2002 international conference, Beijing, China. April 23-27. World Aquaculture Society, 2002.

Gordin H, Krom M, Neori A, Porter C, Shpigel $M$. Seawater mariculture pond. An integrated system. EAS Spec Publ 1981; 11: 45-65.

Hall POJ, Holby O, Kollberg S, Samuelson MO. Chemical fluxes and mass balances in a marine fish cage farm. IV Nitrogen Mar Ecol Prog Ser $1992 ; 89$ : 81-91.

Hargreaves JA. Nitrogen biogeochemistry of aquaculture ponds. Aquaculture 1998; 166 : 181-212. 
Hargreaves JA. Photosynthetic suspendedgrowth systems in aquaculture. Aquac Eng $2006 ; 34: 344-63$.

Heinsbroek LTN, Kamstra A. Design and performance of water recirculation systems for eel culture. Aquac Eng 1990; 9 : 187-207.

Hodges C, Thompson TL, Riley JJ, Glenn EP Reversing the flow: water and nutrients from the sea to the land. Ambio $1993 ; 22: 483-90$.

Holby O, Hall POJ. Chemical fluxes and mass balances in a marine fish cage farm. II Phosphorus. Mar Ecol Prog Ser 1991; 70 : 263-72.

Hussenot J. Les systèmes intégrés en aquaculture marine: une solution durable pour un meilleur respect de l'environnement littoral. In: Chaussade J, Guillaume J, eds. Pêche et aquaculture: pour une exploitation durable des ressources vivantes de la mer et du littoral. Rennes: Presses Universitaires de Rennes, 2006.

Hussenot J, Lefebvre S, Brossard N. Open-air treatment of wastewater from land-based marine fish farms in extensive and intensive systems: current technology and future perspectives. Aquat Living Resour 1998; 11 : 297-304.

Hussenot JME. Emerging effluent management strategies in marine fish-culture farms located in European coastal wetlands. Aquaculture 2003 ; 226 : 113-28.

Hussenot JME, Shpigel M. Marine land-based integrated aquaculture systems for European countries and the $E U$ innovation project "Genesis". In : Chopin T, Reinertsen H, eds. Aquaculture Europe 2003: Beyond monoculture. EAS Special Publication 33. Trondheim, (Norway): European Aquaculture Society (EAS), 2003.

Kamstra A, Van der Heul JW, Nijhof M. Performance and optimisation of trickling filters on eel farms. Aquac Eng 1998 ; 17 : 175-92.

Kumar MS. Proceedings of the National Workshop on Wastewater Treatment and Integrated Aquaculture, South Australian Aquatic Sciences Centre, SARDI, Henley Beach, 2000.

Léonard N, Blancheton JP, Guiraud JP. Populations of heterotrophic bacteria in an experimental recirculating aquaculture system. Aquacult Eng 2000 ; 22 : 109-20.
Léonard N, Guiraud JP, Gasset E, Caillères JP, Blancheton JP. Bacteria and nutrients - nitrogen and carbon - in a recirculating aquaculture system for sea bass production. Aquac Eng $2002 ; 26: 111-27$

Lightfoot C, Bimbao MAP, Dalsgaard JPT, Pullin RSV. Aquaculture and sustainability through integrated resources management. Outlook Agric $1993 ; 22$ : 143-50.

Lüning K, Pang SJ, Garcia Reina G, et al. Species diversification and improvement of aquatic production in seaweeds purifying effluents from integrated fish farms (EU project SEAPURA 2001-2004). In: Chopin T, Reinertsen H, eds. Aquaculture Europe 2003: Beyond monoculture. EAS Special Publication 33. Trondheim (Norway) : European Aquaculture Society (EAS), 2003.

Lupatsch I, Kissil GW. Predicting aquaculture waste from gilthead seabream (Sparus aurata) culture using a nutritional approach. Aquat Living Resour 1998; 11 : 265-8.

Michaud L, Blancheton JP, Bruni V, Piedrahita R. Effect of particulate organic carbon on heterotrophic bacterial populations and nitrification efficiency in biological filters. Aquac Eng 2006 ; 34 : 179-97.

Milstein A. Polyculture in aquaculture. Animal Breeding Abstracts 2005 ; (73): 15N-41N.

Neori A, Chopin T, Troell M, et al. Integrated aquaculture: rationale, evolution and state of the art emphasizing seaweed biofiltration in modern mariculture. Aquaculture 2004; 231 : 361-91.

Pagand P, Blancheton JP, Lemoalle J, Casellas C. The use of high rate algal ponds for the treatment of marine effluent from a recirculating fish rearing system. Aquac Res $2000 ; 31$ : 729-36.

Piedrahita $\mathrm{RH}$. Reducing the potential environmental impact of tank aquaculture effluents through intensification and recirculation. Aquaculture $2003 ; 226$ : 35-44.

Prein M. Integration of aquaculture into cropanimal systems in Asia. Agric Syst 2002; 71 : 127-46.

Rakocy JE. Vegetable hydroponics and fish culture: a productive interface. World Aquac $1989 ; 20: 42-7$.
Romain D. Optimisation du dimensionnement des dispositifs d'amarrage et d'ancrage du modèle de cage aquacole "offshore" utilisé en baie de Saint-Paul (La Réunion). Rapport pour l'obtention du diplôme d'ingénieur Insa de Lyon. SI : ARDA, 2007.

Roque d'Orbcastel E. Optimisation de deux systèmes de production piscicole : biotransformation des nutriments et gestion des rejets. Thèse de doctorat, université Paul-Sabatier, Toulouse, 2008.

Schaber J. FARMSIM: a dynamic model for the simulation of yields, nutrient cycling and resource flows on Philippine small-scale farming systems. Institut für Umweltsystemforschung, University of Osnabrück, 1997.

Schneider O, Sereti V, Eding EH, Verreth JAJ. Analysis of nutrient flows in integrated intensive aquaculture systems. Aquac Eng 2005 $32: 379-401$.

Seawright DE, Stickney RR, Walker RB Nutrient dynamics in integrated aquaculturehydroponics systems. Aquaculture 1998; 160 : 215-37.

Shpigel M, Neori A. The integrated culture of seaweed, abalone, fish and clams in modular intensive land-based systems: I. Proportions of size and projected revenues. Aquac Eng $1996: 15: 313-26$.

Sindilariu PD. Reduction in effluent nutrient loads from flow-through facilities for trout production: a review. Aquac Res 2007; 38 : 1005-36.

Stolte E. Ocean farming. Sustainable exploita tion of the sea. Part: aqua-production park STT document, Den-Haag, Nederlands, 2003.

Verreth JAJ, Eding EH. European farming industry of African catfish (Clarias gariepinus): [facts and figures]. Mag Eur Aquac Soc 1993 $18: 6-13$.

Yan J, Wang R, Wang M. The fundamental principles and ecotechniques of wastewater aquaculture. Ecol Eng 1998 ; 10 : 191-208. 